\title{
Resveratrol alleviates lysophosphatidylcholine-induced damage and inflammation in vascular endothelial cells
}

\author{
JINSONG $\mathrm{CHEN}^{1,2^{*}}$, XIAOCHENG CAO ${ }^{3 *}$, YONGHONG CUI $^{3}$, \\ GAOFENG ZENG $^{2}$, JIAXIAN CHEN ${ }^{2}$ and GUOGANG ZHANG ${ }^{1}$ \\ ${ }^{2}$ Department of Cardiovascular Medicine, The Second Affiliated Hospital of South China University, Hengyang,
}

${ }^{1}$ Department of Cardiovascular Medicine, Xiangya Hospital, Central South University, Kaifu, Changsha, Hunan 410008; Hunan 421001; ${ }^{3}$ Laboratory of Medicine, Medical College, Hunan Normal University, Changsha, Hunan 410016, P.R. China

Received August 2, 2016; Accepted October 26, 2017

DOI: $10.3892 / \mathrm{mmr} .2017 .8300$

\begin{abstract}
The role of resveratrol (trans-3,5,4'-trihydroxystilbene; RES) in lysophosphatidylcholine (LPC)-induced injury and inflammation in endothelial cells (regarded as an early event in arteriosclerosis) is unclear. The present study investigated whether RES reduces lactate dehydrogenase (LDH) activity and secretion of inflammatory cytokines such asinterleukin- 6 and tumor necrosis factor- $\alpha$, via the Toll-like receptor (TLR)-4/myeloid differentiation primary response gene 88 (MyD88)/nuclear factor (NF)- $\kappa \mathrm{B}$ signal transduction pathway in LPC-induced damage and inflammation in human umbilical vein endothelial-12 (HUVE-12) cells. Using an ELISA and western blotting, the present study investigated the effects of RES on LDH activity and cytokine secretion. The effects of TLR-4 short hairpin (sh)RNA and TLR-4 cDNA transfection on $\mathrm{NF}-\kappa \mathrm{B}$ activation during LPC-induced damage and inflammation was also investigated in HUVE-12 cells. The results demonstrated that RES significantly inhibited the effect of LPC on enzyme activity, pro-inflammatory cytokine secretion, and expression of TLR-4, MyD88 and NF- $\mathrm{BP} 65$ expression. In addition, RES and TLR-4 shRNA transfection suppressed LPC-induced injury and inflammation by blocking the TLR-4/MyD88/NF- $\mathrm{B}$ signaling pathway Conversely,
\end{abstract}

Correspondence to: Dr Guogang Zhang, Department of Cardiovascular Medicine, Xiangya Hospital, Central South University, 87 Xiangya Road, Kaifu, Changsha, Hunan 410008, P.R. China

E-mail: xyzgg2006@sina.com

*Contributed equally

Abbreviations: RES, resveratrol; LPC, lysophosphatidylcholine; LDH, lactate dehydrogenase; HUVE-12 cells, human umbilical vein endothelial-12 cells; LDL, low density lipoprotein; TNF- $\alpha$, tumor necrosis factor- $\alpha$; IL-6, interleukin-6; OD, optical density

Key words: resveratrol, lysophosphatidylcholine, endothelial cell, Toll-like receptor-4, myeloid differentiation primary response gene 88 , nuclear factor- $\kappa \mathrm{B}$ transfection with TLR-4 cDNA enhanced LPC-induced injury and inflammation, which abrogated the protective effects of RES. These data suggested that RES significantly suppressed LPC-induced damage and inflammation, via suppression of the TLR-4/MyD88/NF- $\mathrm{B}$ signaling pathway, which may provide a new mechanistic evidence for the treatment of arteriosclerosis by RES.

\section{Introduction}

Inflammation serves a key role in the complex biological response to harmful stimuli in atherosclerosis and coronary heart disease. Lysophosphatidylcholine (LPC), the primary constituent of oxidized low density lipoprotein (LDL), effectively induces oxidative stress in vascular endothelial cells and serves a key etiological role in atherosclerosis (1). LPC is upregulated under inflammatory conditions. In addition, inflammation has a causal association with innate immunity (2), diabetes (3) and cancer (4). It may be attributed to a wide variety of inflammatory cytokines, including tumor necrosis factor- $\alpha$ (TNF- $\alpha$ ), interleukin (IL)-6 and IL-8 (5). Therefore, intervention with an efficacious anti-inflammatory agent is highly desirable in diseases such as atherosclerosis.

Resveratrol (RES) occurs naturally as a polyphenol in various fruits and vegetables and is abundant in grapes (6). Various studies suggest that RES has anticancer, anti-mutation, cardiovascular, anti-thrombotic, anti-microbial, anti-oxidant and immune strengthening activities. Additionally, RES serves a key anti-inflammatory role in diabetes (3), cancer (4), cardiovascular disease (7) and neurodegenerative disease (8).

A previous study demonstrated that the pharmacological activity of RES may be associated with Toll-like receptor (TLR)-4 (9). TLR-4 belongs to the IL-1 receptor (R)/TLR superfamily. It activates the innate immune system (10). In addition, activation of TLR-4 induces NF- $\kappa \mathrm{B}$ expression. The activation of NF- $\kappa \mathrm{B}$ induces the expression of IL-6 and TNF- $\alpha$ (11). However, it is not clear whether RES reduces lactate dehydrogenase (LDH) activity and inflammatory cytokine levels via the TLR-4/MyD88/NF- $\kappa \mathrm{B}$ signaling pathway in LPC-induced damage and inflammation in vitro. Therefore, the present study investigated the effect of TLR-4-mediated NF-KB signaling in the anti-inflammatory response of RES 
to LPC-induced damage and inflammation in the HUVE-12 vascular endothelial cell line.

\section{Materials and methods}

Reagents. RES was purchased from Sigma-Aldrich; Merck KGaA (Darmstadt, Germany; catalog no. R5010). RPMI1640 culture medium was purchased from Gibco (Thermo Fisher Scientific, Inc., Waltham, MA, USA). The lactic acid dehydrogenase assay kit was supplied by Beckman Coulter, Inc. (Brea, CA, USA) and LPC and anti- $\beta$-actin antibodies (cat. no. A4700) were purchased from Sigma-Aldrich; Merck KGaA.

Human TNF- $\alpha$ ELISA (cat. no. BMS223HS) and human IL-6 ELISA (cat. no. EHC007) kits were from NeoBioscience (Shenzhen, China). Anti-TLR-4 (cat. no. AP1504a) and anti-MyD88 (cat. no. 2E9C2) antibodies were from Abgent, Inc. (San Diego, CA, USA). Anti-NF- $\kappa$ B p65 antibodies (cat. no. sc-8008) were provided by Santa Cruz Biotechnology, Inc. (Dallas, TX, USA). OriGene Technologies, Inc. (Rockville, MD, USA) supplied the lentiviral particles packaging of pGFP-V-RS-TLR-4-short hairpin (sh)RNAorpCMV6-AC-GFP-TLR-4-cDNAplasmids.

Cell culture and drug treatment. The human umbilical vein endothelial HUVE-12 cell line was purchased from the Institute of Biochemistry and Cell Biology (Shanghai, China). Cells were cultured at $37^{\circ} \mathrm{C}$ and $5 \% \mathrm{CO}_{2}$ in RPMI-1640 medium (Gibco; Thermo Fisher Scientific, Inc.), supplemented with $10 \%$ heat-inactivated fetal calf serum (FCS; Invitrogen; Thermo Fisher Scientific, Inc.), $100 \mathrm{U} / \mathrm{ml}$ penicillin, and $100 \mu \mathrm{g} / \mathrm{ml}$ streptomycin. HUVE-12 cells stimulated by LPC (final concentrations: 1.0, 10.0 and $100.0 \mu \mathrm{mol} / \mathrm{l}$ ) for $24 \mathrm{~h}$ and DMSO (final concentrations: $0.1 \%$ ) served as the control. In RES treatment experiments, HUVE-12 cells were pretreated with 1,3 and $10 \mu \mathrm{mol} / 1 \mathrm{RES}$ for $2 \mathrm{~h}$ prior to treatment with $10 \mu \mathrm{mol} / 1 \mathrm{LPC}$ for $24 \mathrm{~h}$ at room temperature.

LDH activity. LDH activity was measured and results analyzed using an automated biochemistry analyzer (Beckman Coulter Chemistry analyzer AU-5800 with an Anjue Medical reagent pack, cat no. 1480, Beckman Coulter, Inc., Brea, CA, USA).

ELISA analysis of IL- 6 and TNF- $\alpha$. Levels of TNF- $\alpha$ or IL-6 were quantified in cell supernatant prepared by centrifugation for $5 \mathrm{~min}$ at 2,800 x g using ELISA kits (cat. nos. EHC007 and BMS223HS), according to the manufacturer's protocol. A total of $100 \mu 1$ serially-diluted standard samples or supernatant samples were added to the microplate and incubated at $37^{\circ} \mathrm{C}$ for $1 \mathrm{~h}$. Subsequently, $100 \mu \mathrm{l} 1 \mathrm{X}$ antibody solution against TNF- $\alpha$ or IL- 6 was added to each well and incubated for $37^{\circ} \mathrm{C}$ for $1 \mathrm{~h}$. A total of $100 \mu \mathrm{l}$ horseradish peroxidase-conjugated secondary antibody was added to each well for $30 \mathrm{~min}$ at $37^{\circ} \mathrm{C}$. The plate was washed four times with $100 \mu \mathrm{l}$ PBS containing $0.1 \%$ Tween-20 (PBST) and then the plate was incubated with $100 \mu \mathrm{l} /$ well substrate in the dark for $15 \mathrm{~min}$. The optical density was measured at a wavelength of $450 \mathrm{~nm}$ using a spectrophotometer (Bio-Rad Laboratories, Inc., Hercules, CA, USA).

Cell infection. HUVE-12 cells were seeded onto 24-well plates to a confluence of $40-50 \%$ and incubated overnight at $37^{\circ} \mathrm{C}$.
The cells were infected with lentiviral particles packaging of pGFP-V-RS-TLR-4-shRNA (CCGGCCGCTGGTGTATC TTTGAATACTCGAGTATTCAAAGATACACCAGCGGT TTTTG) or pCMV6-AC-GFP-TLR-4-cDNA (NM_138557) plasmids supplied by OriGene Technologies (Beijing, China) in Opti-MEM (cat. no. 11058-021; Invitrogen; Thermo Fisher Scientific, Inc.) and enhanced infection solution containing $6 \mu \mathrm{g} / \mathrm{ml}$ polybrene (cat. no. REVG0002; Genechem, Inc., Daejeon, Korea). After $4 \mathrm{~h}$, the medium was replaced with RPMI1640 medium containing 10\% FCS. Infected cells were cultured for $48 \mathrm{~h}$ for the assessment of gene expression by western blotting.

Western blotting. Cells were washed with PBS three times and were lysed on ice with radioimmunoprecipitation assay buffer containing $1 \%$ phenylmethylsulfonyl fluoride (Santa Cruz Biotechnology, Inc.). SDS-PAGE (10 or 12\%) was used to separate the proteins in the lysates ( $40 \mu \mathrm{g}$ protein), followed by electroblotting of the proteins onto a polyvinylidene difluoride membrane (EMD Millipore, Billerica, MA, USA). Membranes were blocked in 5\% non-fat milk in PBST for $1 \mathrm{~h}$ at room temperature, and probed with the following mouse anti-human primary monoclonal antibodies: Anti-TLR-4 $(1: 1,000)$, anti-MyD88 (1:1,000), anti-NF-кBp65 (1:1,000) and anti- $\beta$-actin $(1: 2,000)$, under slight vibration at $4^{\circ} \mathrm{C}$ overnight. Membranes were subsequently incubated with a goat polyclonal secondary antibody (cat. no. 31430; 1:500; Invitrogen; Thermo Fisher Scientific, Inc.) to mouse immunoglobulin Gse immunoglobulin $\mathrm{G}$ for $1 \mathrm{~h}$ at room temperature, followed by an Enhanced Chemiluminescence substrate solution (GE Healthcare; Chicago, IL, USA).

Statistical analysis. Data are expressed as the mean \pm standard deviation. SPSS software version 15.0 (SPSS, Inc., Chicago, IL, USA) was used for statistical analysis and a one-way analysis of variance followed by Tukey's post-hoc test was used to analyze significant differences in mean values. $\mathrm{P}<0.05$ was considered to indicate a statistically significant difference.

\section{Results and Discussion}

Effects of LPC on LDH activity, IL-6 and TNF-asecretion, and TLR-4, MyD88 and NF- $\mathrm{B}$ expression in HUVE-12 cells. Treatment of HUVE-12 cells with 10 and $100 \mu \mathrm{mol} / 1 \mathrm{LPC}$ significantly enhanced LDH activity and the levels of IL-6 and TNF- $\alpha$ compared with cells treated with $0.1 \%$ DMSO (0 $\mu \mathrm{mol} / 1$ LPS; $>0.01$; Fig. $1 \mathrm{~A}-\mathrm{C})$, which suggested that LPC induced damage and inflammation. In addition, treatment with 1,10 and $100 \mu \mathrm{mol} / 1 \mathrm{LPC}$ elevated the expression levels of TLR-4, MyD88 and NF- $\mathrm{B}$ p65 in HUVE-12 cells in a concentration-dependent manner compared with cells treated with $0 \mu \mathrm{mol} / 1$ LPC (Fig. 1D-F). These results suggested that TLR-4 signaling mediates HUVE-12 cell injury induced by LPC. LDH is a marker of injury and diseases, including heart failure (12). Oxidation and enzymatic modification of low density lipoprotein (LDL) leads to lysophosphatidylcholine (LPC) synthesis in atherosclerosis (12). LPC induces inflammation in coronary artery smooth muscle cells (13). Qin et al (14) demonstrated that LPC maintains macrophage polarization towards a classically activated phenotype in inflammation. 
A

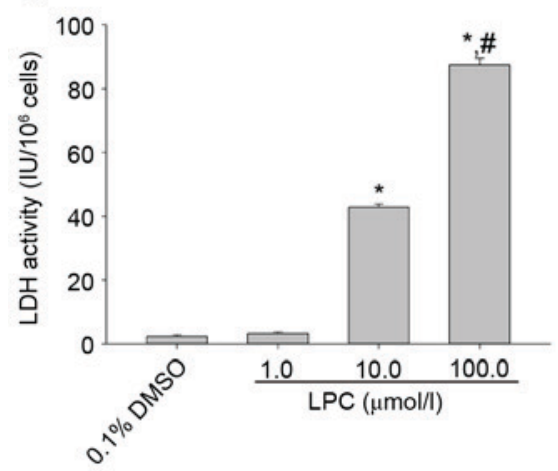

D

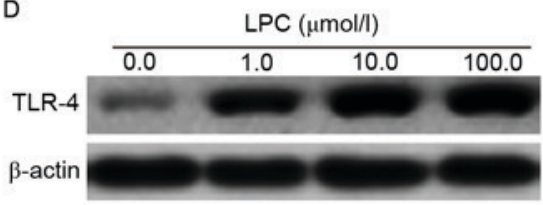

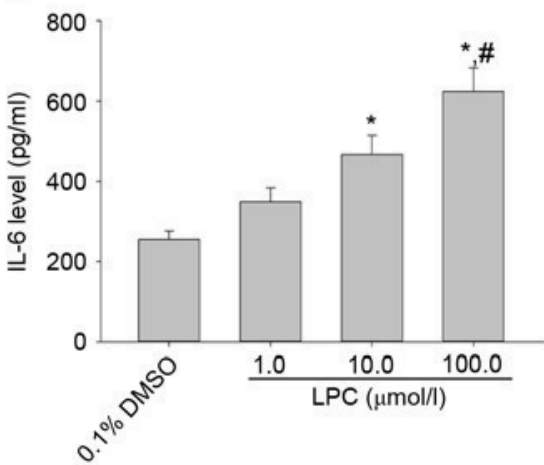

$\mathrm{E}$

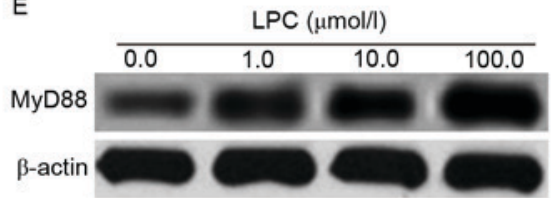

C

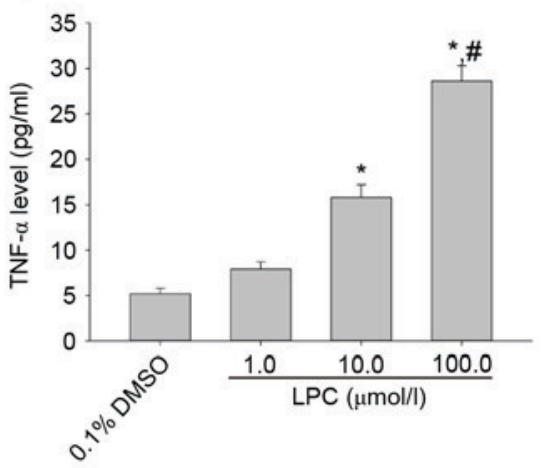

F

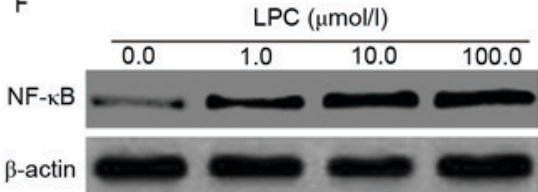

Figure 1. LPC induces inflammation and activates the TLR-4/MyD88/NF- $\mathrm{B}$ signaling pathway in HUVE-12 cells. (A) Activity of LDH and secretion of (B) IL-6 and (C) TNF- $\alpha$ were elevated after treatment with 10.0 and $100.0 \mu \mathrm{M} \mathrm{LPC}$. Data are expressed as the mean \pm standard deviation. ${ }^{*} \mathrm{P}<0.05 \mathrm{vs} .0 \mu \mathrm{mol} / 1$ (0.1\% DMSO) and ${ }^{\#} \mathrm{P}<0.05$ vs. $1.0 \mu \mathrm{mol} / 1 \mathrm{LPC}$. In addition, 1.0, 10.0 and $100.0 \mu \mathrm{M}$ LPC treatment resulted in increased levels of (D) TLR-4, (E) MyD88 and

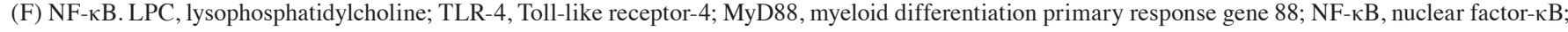
LDH, lactate dehydrogenase; IL-6, interleukin-6; TNF- $\alpha$, tumor necrosis factor- $\alpha$.

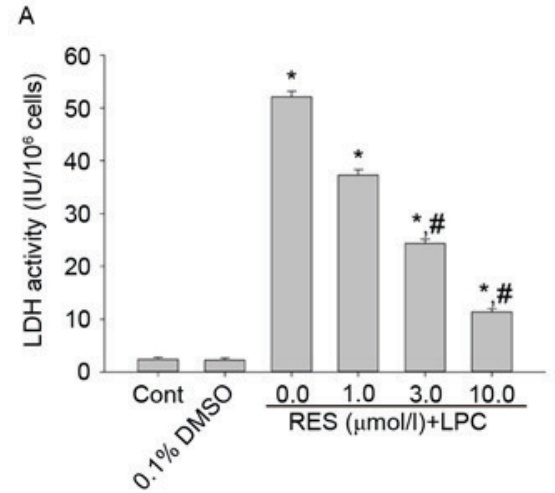

D

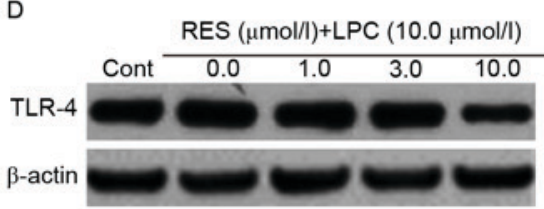

B

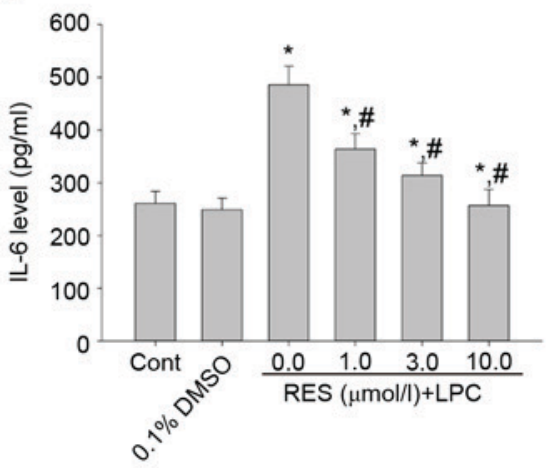

$\mathrm{E}$

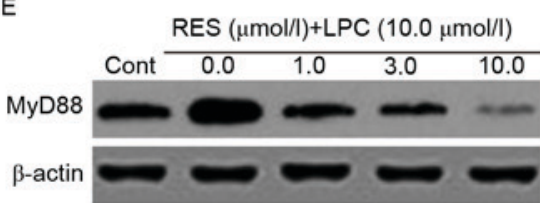

C

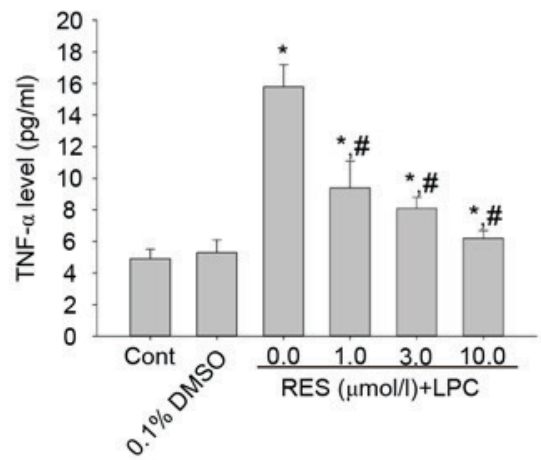

$\mathrm{F}$

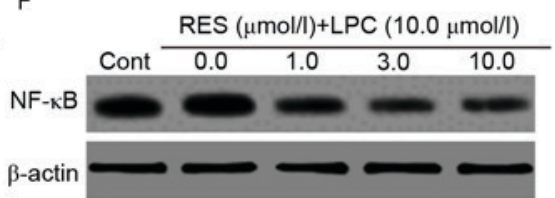

Figure 2. Pretreatment with RES suppresses the inflammation induced by the subcytotoxic concentration of $10.0 \mu \mathrm{mol} / 1 \mathrm{LPC}$ and decreases activation of the TLR-4/MyD88/NF-kB signaling pathway in HUVE-12 cells. The effect of LPC on (A) LDH activity, (B) IL-6 and (C) TNF- $\alpha$ was suppressed by 1.0, 3.0 and 10.0 $\mu \mathrm{M}$ RES in HUVE-12 cells. Furthermore, LPC-induced upregulation of (D) TLR-4 (E) MyD88 and (F) NF- $\mathrm{kB}$ was suppressed by RES treatment. Data are expressed as the mean \pm standard deviation. ${ }^{*} \mathrm{P}<0.05 \mathrm{vs} .0 \mu \mathrm{mol} / 1(0.1 \% \mathrm{DMSO}) ;{ }^{*} \mathrm{P}<0.05 \mathrm{vs} .0 .0 \mu \mathrm{mol} / 1$ RES. Cont, untreated HUVE-12 cells; RES,

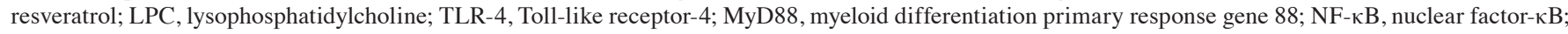
LDH, lactate dehydrogenase; IL-6, interleukin-6; TNF- $\alpha$, tumor necrosis factor- $\alpha$.

Li et al (15) reported that LPC induces the secretion of inflammatory factors in human umbilical vein endothelial cells. The results of the present study suggested that LPC increased LDH activity and expression of the inflammatory cytokines, IL-6 and TNF- $\alpha$, suggesting that LPC induced injury and inflammation in HUVE-12 cells.

Pathogens, cytokines and environmental stimuli alter TLR-4 expression in vascular injury and the inflammatory response. TLRs mediate zinc/nickel-induced inflammation in endothelial cells (16). Wang et al (17) reported that TLR-4 stimulates proliferation and an inflammatory response in LPS-induced Hep G2 cells. Bomfim et al (18) suggested that TLR-4 mediates hypertension and vascular inflammation via NF- $\kappa \mathrm{B}$ signaling. The interaction between TLR-4 and proteinase-activated receptor 2 [PAR (2)] contributes to vascular homeostasis (19). The present study demonstrated that TLR-4 signaling may be involved in LPC-induced injury and inflammation in HUVE-12 cells. 

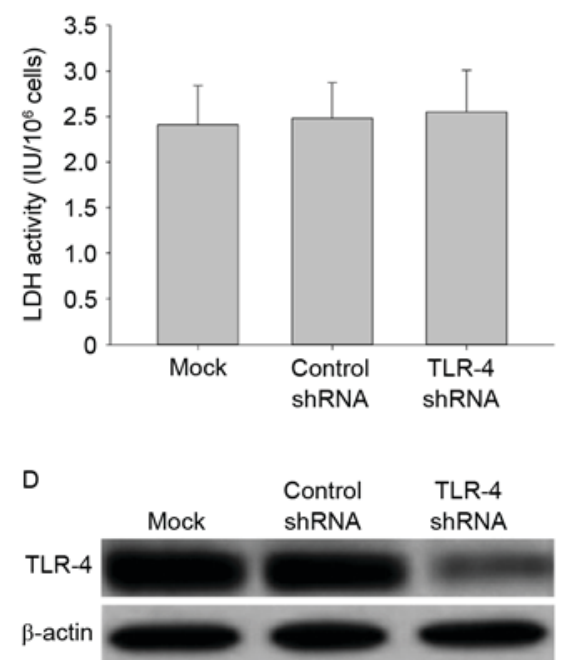

$B$

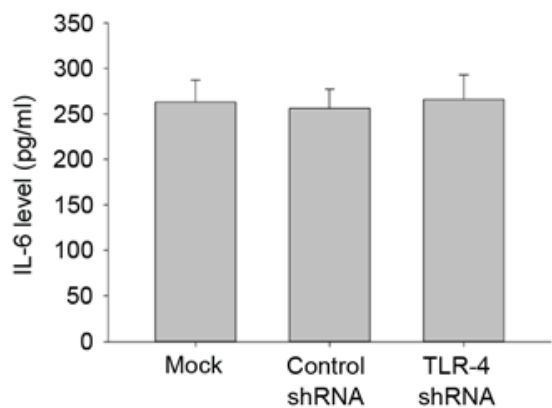

$\mathrm{E}$

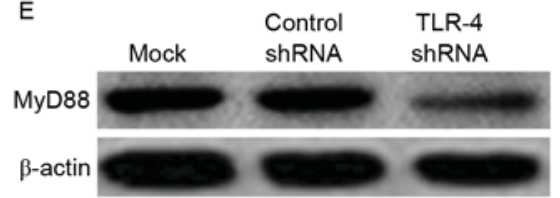

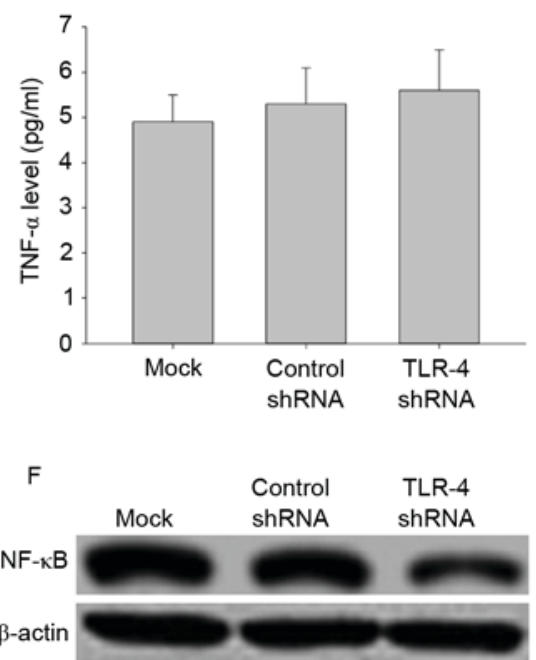

Figure 3. Transfection with TLR-4 shRNA blocks TLR-4/MyD88/NF- $\mathrm{BB}$ signaling in HUVE-12 cells. (A) TLR-4-shRNA transfection did not affect LDH activity. Knockdown of TLR-4 had no effect on the levels of (B) IL-6 or (C) TNF- $\alpha$. However, the expression of (D) TLR-4, (E) MyD88 and (F) NF- $\mathrm{kB}$ were decreased following TLR-4 shRNA transfection. Data are expressed as the mean \pm standard deviation. TLR-4, Toll-like receptor-4; shRNA, short hairpin

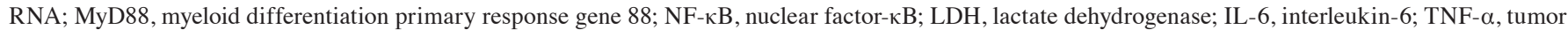
necrosis factor- $\alpha$.
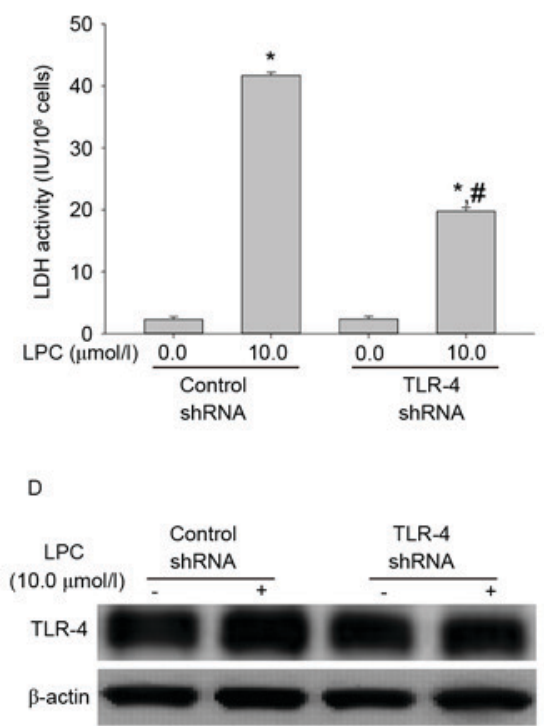
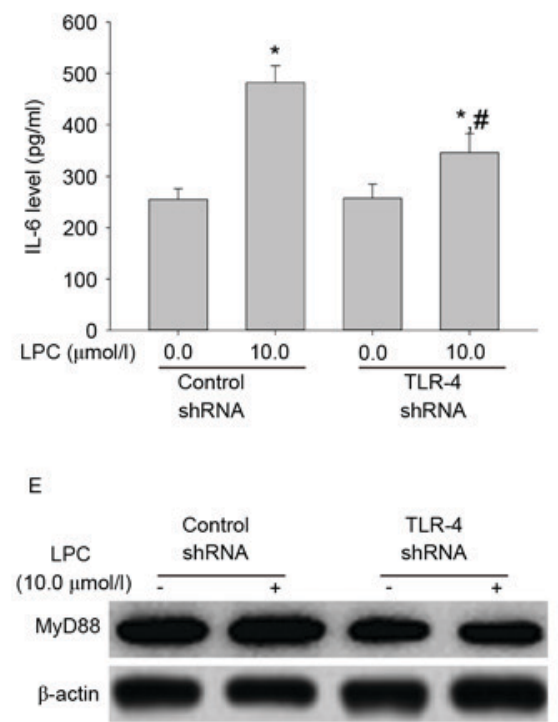

C

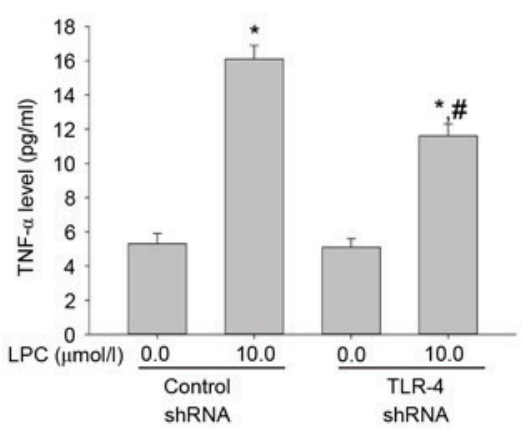

$\mathrm{F}$

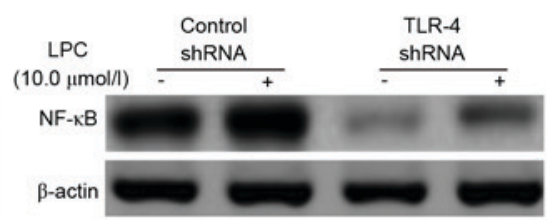

Figure 4. TLR-4 shRNA transfection inhibits LPC-induced damage and inflammation. (A) Compared with control shRNA, transfection with TLR-4 shRNA suppressed LPC-induced activity of LDH. (B) TLR-4 shRNA inhibited the effects of LPC on secretion of IL- 6 and (C) TNF- $\alpha$. Furthermore, TLR-4shRNA downregulated LPC-induced expression of (D) TLR-4, (E) MyD88 and (F) NF- $\kappa$ B. Data are expressed as the mean \pm standard deviation. "P $<0.01$ vs. control shRNA in absence of $10.0 \mu \mathrm{mol} / 1 \mathrm{LPC}$; ${ }^{\mathrm{P}} \mathrm{P}<0.05 \mathrm{vs}$. control shRNA in presence of $10.0 \mu \mathrm{mol} / 1 \mathrm{LPC}$. TLR-4, Toll-like receptor-4; shRNA, short hairpin

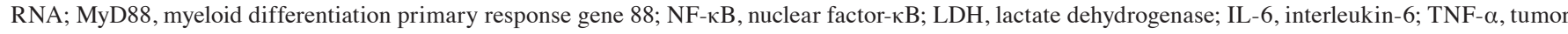
necrosis factor- $\alpha$; LPC, lysophosphatidylcholine.

Effect of RES on LDH activity, IL-6 and TNF-asecretion, and TLR-4, MyD88 and NF- $\kappa B$ p65 expression. To examine whether RES protects against LPC-induced injury and inflammation, HUVE-12 cells were pretreated with 1, 3 and $10 \mu \mathrm{mol} / 1 \mathrm{RES}$ prior to treatment with $10 \mu \mathrm{mol} / \mathrm{l}$ LPC. RES inhibited the effects of LPC on LDH activity and cytokine expression compared with cells treated with $0 \mu \mathrm{mol} / 1 \mathrm{RES}$ and LPC $(\mathrm{P}<0.01$; Fig. 2A-C). In addition, RES suppressed the expression levels of TLR-4, MyD88 and NF- $\kappa \mathrm{B}$ compared with cells treated with $0 \mu \mathrm{mol} / \mathrm{l} \mathrm{RES}$ (Fig. 2D-F), which were upregulated by LPC treatment alone. RES has been reported to exhibit anti-atherogenic effects (20). Various studies suggested that RES protects cardiomyocytes against injury via the TLR-4/NF- $\mathrm{B}$ signaling pathway $(21,22)$. The results of the present study revealed that RES may protect from the LPC-induced damage and inflammation by inhibiting the TLR-4/MyD88/NF-кB signaling pathway. 

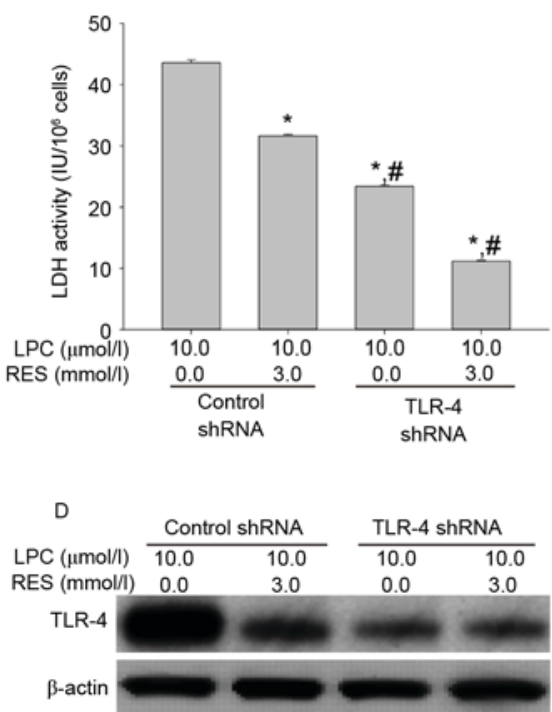

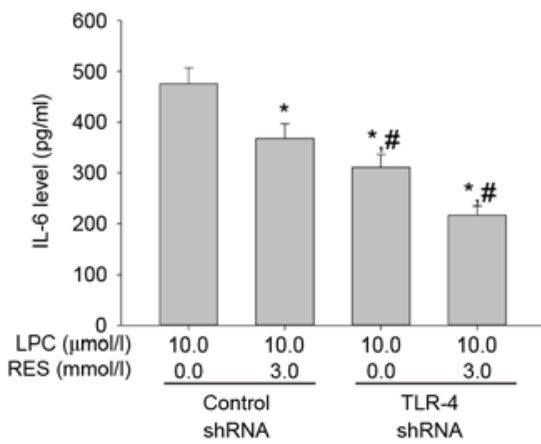

$E$

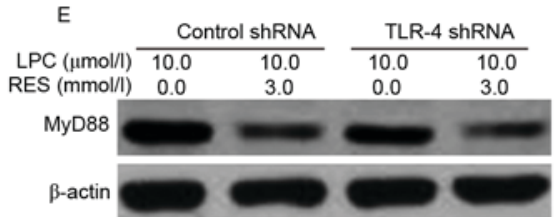

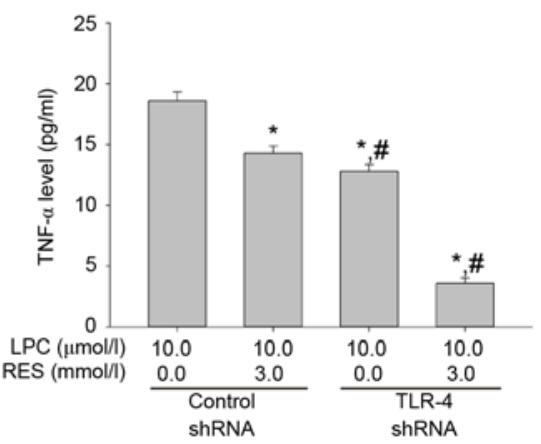

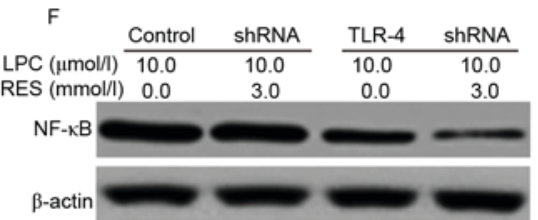

Figure 5. RES and transfection with TLR-4 shRNA cooperatively suppress LPC-induced inflammation by blocking the TLR-4/MyD88/NF- $\kappa B$ signaling pathway in HUVE-12 cells. (A) RES and TLR-4-shRNA transfection suppressed LPC-induced activity of LDH. RES and TLR-4 shRNA transfection suppressed LPC-increased cytokine expression of (B) IL-6 and (C) TNF- $\alpha$. In addition, RES and TLR-4-shRNA transduction suppressed LPC-mediated

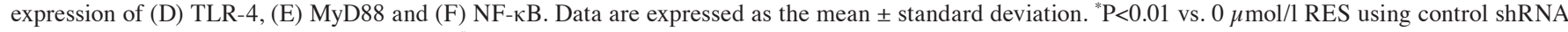
transfection in presence of $10.0 \mu \mathrm{mol} / 1 \mathrm{LPC} ;{ }^{~} \mathrm{P}<0.05 \mathrm{vs} .3 .0 \mu \mathrm{mol} / 1 \mathrm{RES}$ using control shRNA transfection in presence of $10.0 \mu \mathrm{mol} / 1 \mathrm{LPC}$. RES, resveratrol;

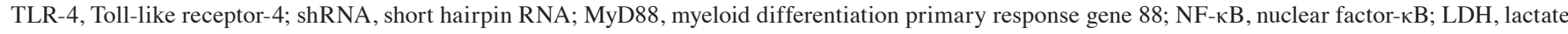
dehydrogenase; IL-6, interleukin-6; TNF- $\alpha$, tumor necrosis factor- $\alpha$; LPC, lysophosphatidylcholine.

A

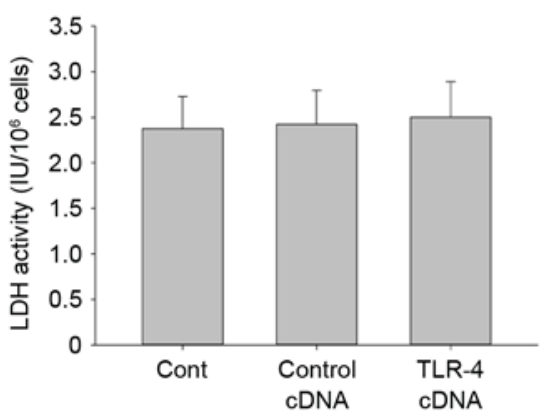

D

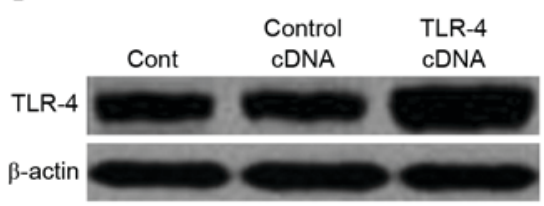

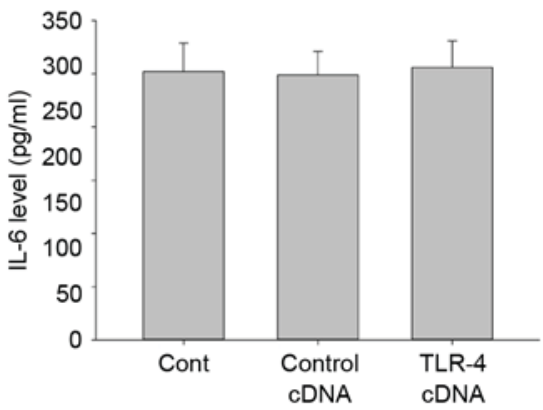

$\mathrm{E}$

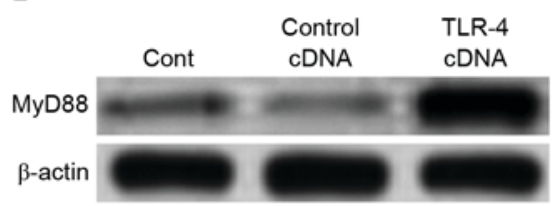

C
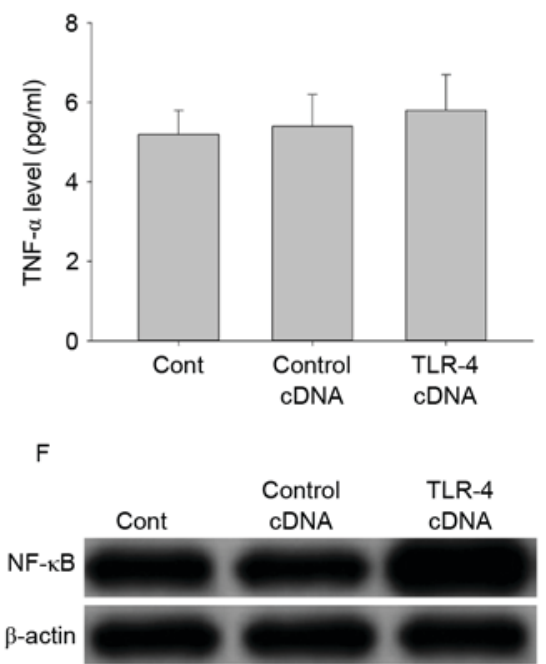

Figure 6. Transfection with TLR-4 cDNA activates the TLR-4/MyD88/NF-kB signaling pathway in HUVE-12 cells. (A) Transfection with TLR-4 cDNA did not affect LDH activity or expression of (B) IL-6 or (C) TNF- $\alpha$. However, TLR-4 cDNA visibly enhanced the expression of (D) TLR-4, (E) MyD88 and (F) NF-kB compared with Cont or Control cDNA groups. Data are expressed as the mean \pm standard deviation. Cont, untreated HUVE-12 cells; Contrl cDNA, GFP cDNA transduced HUVE-12 cells; TLR-4, Toll-like receptor-4; MyD88, myeloid differentiation primary response gene 88; NF- $\mathrm{B}$, nuclear factor- $\mathrm{kB}$; LDH, lactate dehydrogenase; IL-6, interleukin-6; TNF- $\alpha$, tumor necrosis factor- $\alpha$.

Effects of TLR-4 ShRNA transfection on LDH activity, $I L-6$ and TNF-asecretion, and signal transduction. To further investigate the role of TLR-4 in LPC-induced damage and inflammation in HUVE-12 cells, TLR-4 shRNA transduction was performed to silence the TLR-4 gene. Transfection with TLR-4-shRNA did not affect LDH activity or expression of IL- 6 and TNF- $\alpha$ in HUVE-12 cells (Fig. 3A-C), despite demonstrating that transfection with TLR-4-shRNA significantly decreased the expression levels of TLR-4 and its downstream targets, MyD88 and NF- $\mathrm{KB}$, compared with cells transfected with control shRNA (Fig. 3D-F). These results suggested that knockdown of TLR- 4 silenced the TLR- 4 gene to inhibit the TLR-4/MyD88/NF- $\mathrm{BB}$ signaling pathway; while it had little effect on injury and inflammatory factor secretion of HUVE-12 cells. 
A

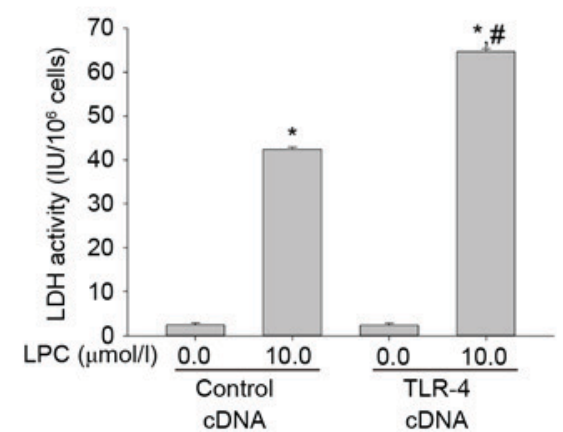

D

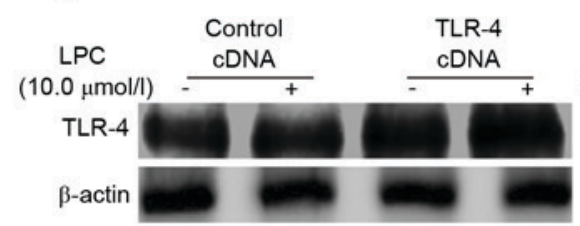

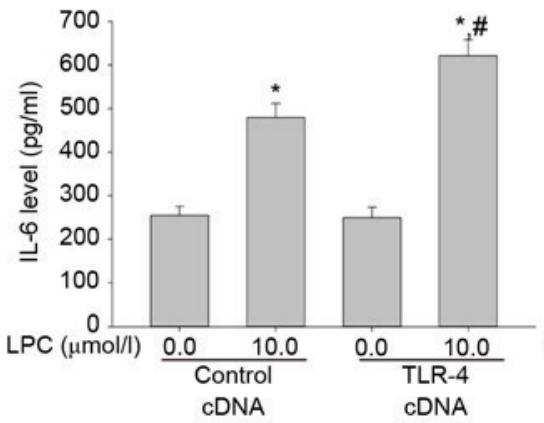

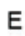

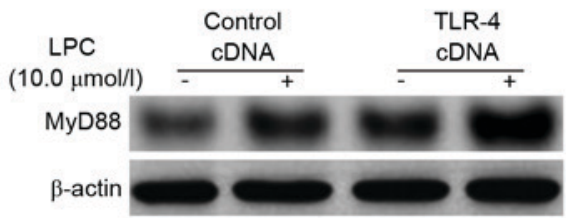

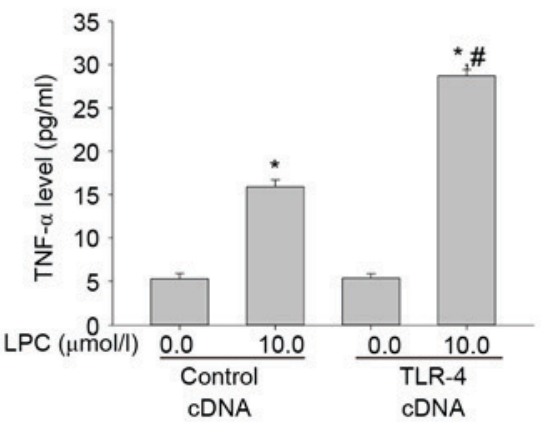

$\mathrm{F}$

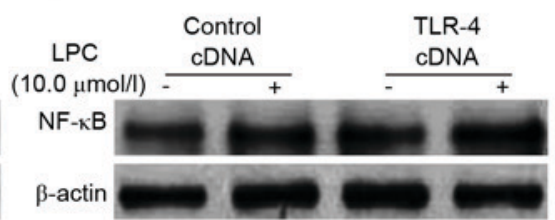

Figure 7. TLR-4 cDNA transfection increases LPC-induced damage and inflammation. (A) Compared with control cDNA, the increased activity of LDH by LPC was elevated after transfection with TLR-4 cDNA. TLR-4 cDNA transduction increased the effect of LPC on (B) IL-6 and (C) TNF- $\alpha$ expression. Furthermore, the levels of (D) TLR-4, (E) MyD88 and (F) NF- $\kappa$ B increased following TLR-4 cDNA transduction compared with LPC-stimulated HUVE-12 cells transduced with GFP cDNA. ${ }^{*} \mathrm{P}<0.01$ vs. control cDNA in the absence of $10.0 \mu \mathrm{mol} / 1 \mathrm{LPC} ;{ }^{\#} \mathrm{P}<0.05 \mathrm{vs}$. control cDNA in the presence of $10.0 \mu \mathrm{mol} / 1 \mathrm{LPC}$. Data are expressed as the mean \pm standard deviation. TLR-4, Toll-like receptor-4; MyD88, myeloid differentiation primary response gene 88 ; NF- $\kappa$ B, nuclear factor- $\kappa \mathrm{B}$; LDH, lactate dehydrogenase; IL-6, interleukin-6; TNF- $\alpha$, tumor necrosis factor- $\alpha$; LPC, lysophosphatidylcholine.

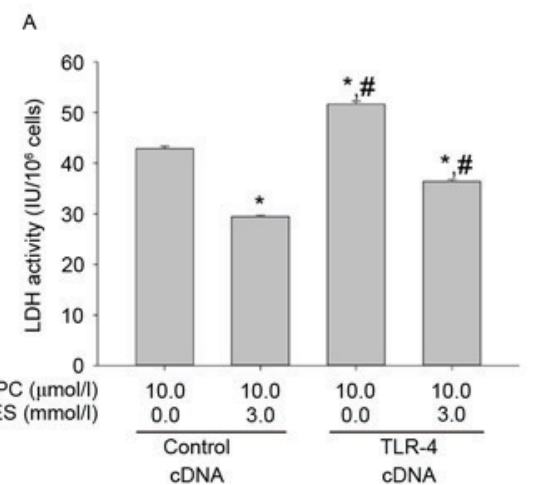

D

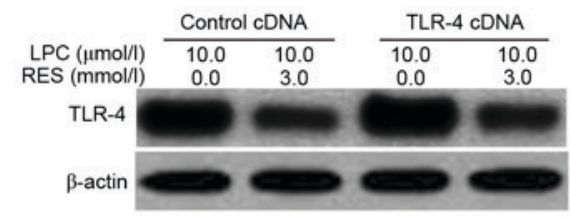

B

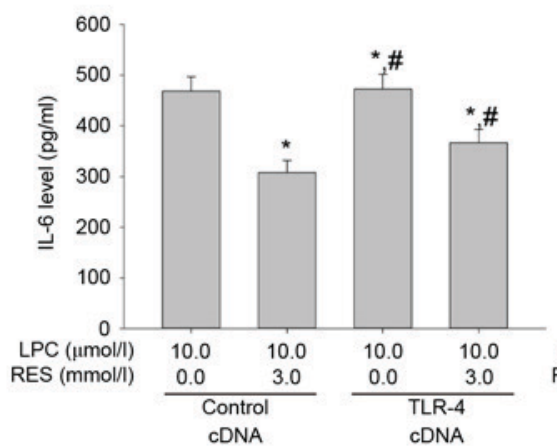

$E$

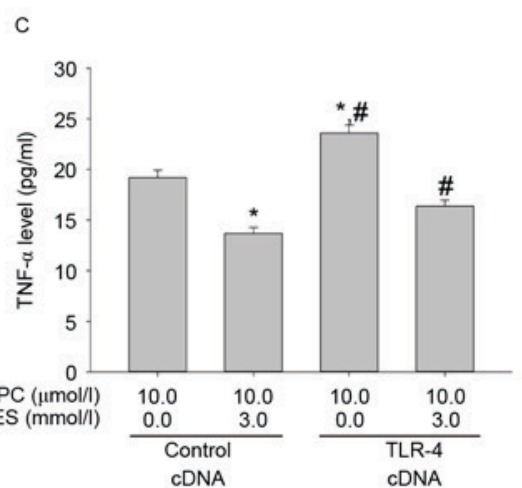

$\mathrm{F}$

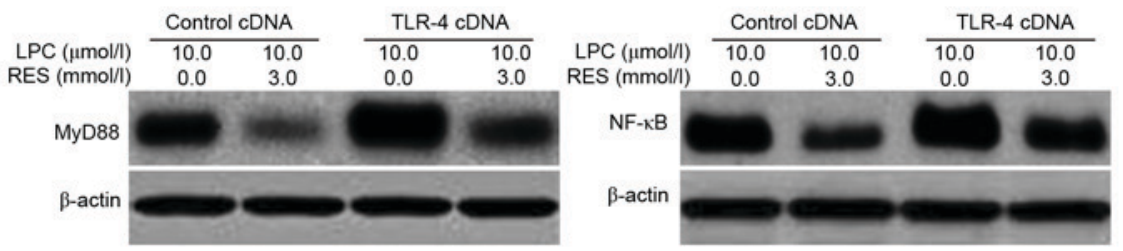

Figure 8. Transfection with TLR-4 cDNA antagonizes the inhibitory effects of RES on LPC-induced inflammation by activating the NF- $\kappa$ B signaling pathway in HUVE-12 cells. (A) Inhibition of LPC-induced LDH activity by RES was antagonized by TLR-4 cDNA transduction. The reduction in (B) IL-6 and (C) TNF- $\alpha$ by RES in the presence of LPC was attenuated by TLR-4 cDNA transfection. Furthermore, the downregulation inexpression of (D) TLR-4, (E) MyD88 and (F) NF- $\mathrm{B}$ by RES in the presence of LPC was reversed following transfection with TLR-4 cDNA. ${ }^{*}<0.01$ vs. control cDNA in absence of $3.0 \mu \mathrm{mol} / 1 \mathrm{RES} ;{ }^{\#} \mathrm{P}<0.05$ vs. control cDNA in presence of $3.0 \mu \mathrm{mol} / 1 \mathrm{RES}$. Data are expressed as the mean \pm standard deviation. TLR-4, Toll-like receptor-4; MyD88, myeloid differentiation primary response gene 88 ; NF- $\kappa \mathrm{B}$, nuclear factor- $\kappa \mathrm{B}$; LDH, lactate dehydrogenase; IL-6, interleukin-6; TNF- $\alpha$, tumor necrosis factor- $\alpha$; LPC, lysophosphatidylcholine; RES, resveratrol.

TLR-4 shRNA transfection influences LDH activity, IL-6 and TNF-asecretion, and signal transduction induced by LPC treatment. To evaluate the effect of TLR-4 shRNA on LPC-induced damage and inflammation, the LDH activity and levels of IL- 6 and TNF- $\alpha$ were measured. As demonstrated in Fig. 4A-C, transfection with TLR-4 shRNA significantly inhibited the effects of LPC on LDH activity and IL-6 and TNF- $\alpha$ cytokine secretion, compared with control shRNA $(\mathrm{P}<0.01)$. Furthermore, TLR-4 shRNA suppressed LPC-induced upregulation of signaling molecules compared with control shRNA (Fig. 4D-F). This suggested that TLR-4 gene silencing may protect from LPC-induced damage and inflammation. 
Effects of RES therapy and TLR-4 shRNA transfection on LDH activity, IL- 6 and TNF-asecretion, and TLR-4, MyD88 and $N F-\kappa B$ expression. To investigate whether RES inhibits LPC-induced damage and inflammation in human umbilical vein endothelial cells, the effect of RES in combination with TLR-4 shRNA on LPC-induced damage and inflammation in HUVE-12 cells was investigated. RES treatment and TLR-4 shRNA transfection suppressed the effects of LPC on LDH activity and IL-6 and TNF- $\alpha$ secretion ( $\mathrm{P}<0.01$; Fig. 5A-C), the expression of TLR-4 and MyD88 were weakly downregulated, and the expression of $\mathrm{NF}-\kappa \mathrm{B}$ were markedly downregulated (Fig. 5D-F). These data suggested that RES inhibited expression of NF- $\mathrm{B}$ may have involved another mechanism.

A previous study demonstrated that the pharmacological activity of RES may be associated with TLR-4. The interaction of TLR-4 with MyD88 activates TNF receptor-associated factor, to activate the inflammatory cascade (23). TLR-4 may mediate MyD88-dependent NF- $\kappa \mathrm{B}$ activation, which increases the production of inflammatory cytokines. The results of the present study suggested that RES attenuates LPC-induced damage and inflammation in HUVE-12 cells by inhibiting the TLR-4/MyD88/NF- $\mathrm{B}$ signaling pathway.

Effect of TLR-4 overexpression on LDH activity, IL-6 and $T N F$-asecretion, and signal transduction. To further investigate the role of TLR-4 in LPC-induced damage and inflammation in HUVE-12 cells, cells were transfected with TLR-4 cDNA to overexpress the TLR-4 gene. As demonstrated in Fig. 6A-C, no significant differences were observed in LDH activity and cytokine expression between cells transfected with the control cDNA and TLR-4 cDNA ( $>0.05)$. However, the expression levels of TLR-4 and its downstream proteins including MyD88 and $\mathrm{NF}-\kappa \mathrm{B}$, were elevated by TLR-4 overexpression (Fig. 6D-F). These results suggested that overexpression of TLR-4 may activate the TLR-4/MyD88/NF-кB signaling pathway.

Effect of TLR-4 overexpression on LDH activity, IL-6 and $T N F$-asecretion, and signal transduction induced by LPC. To determine the effect of TLR-4 overexpression on LPC-induced damage and inflammation, the LDH activity and levels of IL-6 and TNF- $\alpha$ were measured. Transfection with TLR-4 cDNA enhanced LPC-induced LDH activity and IL-6 and TNF- $\alpha$ levels compared with cells transfected with control cDNA in HUVE-12 cells ( $\mathrm{P}<0.01$; Fig. 7A-C). The expression levels of TLR-4, MyD88 and NF- $\kappa$ B in cells transfected with TLR cDNA was higher than in the control cDNA group after LPC treatment (Fig. 7D-F), which suggested that TLR-4 may be associated with $\mathrm{NF}-\kappa \mathrm{B}$ signaling during LPC-induced damage and inflammation (18).

Effects of RES treatment combined with TLR-4 cDNA transduction on LDH activity, IL-6 and TNF-asecretion, and signal transduction. To confirm that the effects of RES inhibited LPC-induced damage and inflammation in human umbilical vein endothelial cells via regulation of the TLR-4/MyD88/NF- $\kappa$ B signaling pathway, the effect of RES treatment and TLP4 overexpression on LPC-induced damage and inflammation in HUVE-12 cells was investigated. RES significantly suppressed the effects of TLR-4 overexpression on LPC-induced damage and inflammation ( $\mathrm{P}<0.01$; Fig. 8A-F).
RES was reported to exhibit anti-atherogenic effects (20). Various studies suggested that RES protects cardiomyocytes against injury via the TLR-4/NF- $\kappa \mathrm{B}$ signaling pathway $(21,22)$. The results of the present study supported the critical role served by TLR-4/MyD88/NF- $\mathrm{B}$ signaling in LPC-induced injury and pro-inflammatory responses. In addition, the present study demonstrated that RES attenuated the inflammatory reaction induced by LPC in HUVE-12 cells via downregulation of the TLR-4/MyD88/NF- $\mathrm{B}$ signaling pathway. The present study reported that RES exercises protective actions in the first steps of the atherogenic process. Reducing the expression of adhesion molecules (intercellular adhesion molecule-1, and vascular cell adhesion molecule-1) via inhibition of NF- $\kappa \mathrm{B}$ pathway activation by RES was demonstrated by Deng et al (24). The present study also provided evidence that RES inhibited $\mathrm{NF}-\kappa \mathrm{B}$ activation through blocking TLR-4/MyD88/NF- $\mathrm{B}$ signal pathway. The results highlight the anti-inflammatory properties and potential molecule mechanism of RES. Bonnefont-Rousselot (25) suggested that RES is a good candidate, owing to its protective action of vascular walls towards oxidation, inflammation, platelet oxidation and thrombus formation. RES may be beneficial in preventing the development of atherosclerosis. However, further studies with animal models are required to validate the findings of the present study.

\section{References}

1. Domeij H, Hua X, Su J, Bäcklund A, Yan Z, Frostegard AG, Haeggström JZ, Modéer T and Frostegard J: Annexin A5 inhibits atherogenic and pro-inflammatory effects of lysophosphatidylcholine. Prostaglandins Other Lipid Mediat 106: 72-78, 2013.

2. Sheldon IM, Cronin JG, Healey GD, Gabler C, Heuwieser W, Streyl D, Bromfield JJ, Miyamoto A, Fergani C and Dobson H: Innate immunity and inflammation of the bovine female reproductive tract in health and disease. Reproduction 148: R41-R51, 2014.

3. Gao W, Zhou Y, Li Q, Zhou Q, Tan L, Song Y, Zhao X, Yu M, Zheng S, Ye H, et al: Analysis of global gene expression profiles suggests a role of acute inflammation in type $3 \mathrm{C}$ diabetes mellitus caused by pancreatic ductal adenocarcinoma. Diabetologia 58: 835-844, 2015.

4. Coussens LM and Werb Z: Inflammation and cancer. Nature 420: 860-867, 2002

5. Strowig T, Henao-Mejia J, Elinav E and Flavell R: Inflammasomes in health and disease. Nature 481: 278-286, 2012.

6. Bradamante S, Barenghi L and Villa A: Cardiovascular protective effects of resveratrol. Cardiovasc Drug Rev 22: 169-188, 2004.

7. Libby P, Ridker PM and Maseri A: Inflammation and atherosclerosis. Circulation 105: 1135-1143, 2002.

8. Kim D, Nguyen MD, Dobbin MM, Fischer A, Sananbenesi F, Rodgers JT, Delalle I, Baur JA, Sui G, Armour SM, et al: SIRT1 deacetylase protects against neurodegeneration in models for Alzheimer's disease and amyotrophic lateral sclerosis. EMBO J 26: 3169-3179, 2007.

9. Youn HS, Lee JY, Fitzgerald KA, Young HA, Akira S and Hwang DH: Specific inhibition of MyD88-independent signaling pathways of TLR3 and TLR4 by resveratrol: Molecular targets are TBK1 and RIP1 in TRIF complex. J Immunol 175: 3339-3346, 2005.

10. Apetoh L, Ghiringhelli F, Tesniere A, Obeid M, Ortiz C, Criollo A, Mignot G, Maiuri MC, Ullrich E, Saulnier P, et al: Toll-like receptor 4-dependent contribution of the immune system to anticancer chemotherapy and radiotherapy. Nat Med 13: 1050-1059, 2007.

11. Huang RL, Yuan Y,Zou GM,Liu G, Tu J and Li Q: LPS-stimulated inflammatory environment inhibits BMP-2-induced osteoblastic differentiation through crosstalk between TLR4/MyD88/NF-kB and BMP/Smad signaling. Stem Cells Dev 23: 277-289, 2014. 
12. Augoff K, Hryniewicz-Jankowska A and Tabola R: Lactate dehydrogenase 5: An old friend and a new hope in the war on cancer. Cancer Lett 358: 1-7, 2015.

13. Aiyar N, Disa J, AoZ, Ju H, Nerurkar S, Willette RN, Macphee CH, Johns DG and Douglas SA: Lysophosphatidylcholine induces inflammatory activation of human coronary artery smooth muscle cells. Mol Cell Biochem 295: 113-120, 2007.

14. Qin X, Qiu C and Zhao L: Lysophosphatidylcholine perpetuates macrophage polarization toward classically activated phenotype in inflammation. Cell Immunol 289: 185-190, 2014.

15. Li JZ, Wu JH, Yu SY, Shao QR and Dong XM: Inhibitory effects of paeoniflorin on lysophosphatidylcholine-induced inflammatory factor production in human umbilical vein endothelial cells. Int J Mol Med 31: 493-497, 2013.

16. Tsou TC, Liou SH, Yeh SC, Tsai FY and Chao HR: Crucial role of Toll-like receptors in the zinc/nickel-induced inflammatory response in vascular endothelial cells. Toxicol Appl Pharmacol 273: 492-499, 2013.

17. Wang Y, Tu Q, Yan W, Xiao D, Zeng Z, Ouyang Y, Huang L, Cai J, Zeng X, Chen YJ and Liu A: CXC195 suppresses proliferation and inflammatory response in LPS-induced human hepatocellular carcinoma cells via regulating TLR4-MyD88-TAK1-mediated NF-kB and MAPK pathway. Biochem Biophys Res Commun 456 373-379, 2015

18. Bomfim GF, Echem C, Martins CB, Costa TJ, Sartoretto SM, Dos Santos RA, Oliveira MA, Akamine EH, Fortes ZB, Tostes RC, et al: Toll-like receptor 4 inhibition reduces vascular inflammation in spontaneously hypertensive rats. Life Sci 122 : $1-7,2015$.
19. Bucci M, Vellecco V, Harrington L, Brancaleone V, Roviezzo F, Mattace Raso G, Ianaro A, Lungarella G, De Palma R, Meli R and Cirino G: Cross-talk between Toll-like receptor 4 (TLR4) and proteinase-activated receptor 2 (PAR (2)) is involved in vascular function. Br J Pharmacol 168: 411-420, 2013.

20. Riccioni G, Gammone MA, Tettamanti G, Bergante S, Pluchinotta FR and D'Orazio N: Resveratrol and anti-atherogenic effects. Int J Food Sci Nutr 66: 603-610, 2015.

21. Zhang C, Lin G, Wan W, Li X, Zeng B, Yang B and Huang C: Resveratrol, a polyphenol phytoalexin, protects cardiomyocytes against anoxia/reoxygenation injury via the TLR4/NF- $\mathrm{B}$ signaling pathway. Int J Mol Med 29: 557-563, 2012.

22. Li J, Xie C, Zhuang J, Li H, Yao Y, Shao C and Wang H: Resveratrol attenuatesinflammationintheratheartsubjected toischemia-reperfusion: Role of the TLR4/NF-кB signaling pathway. Mol Med Rep 11: 1120-1126, 2015.

23. Feng $\mathrm{Y}$ and Longmore GD: The LIM protein Ajuba influences interleukin-1-induced NF-kappaB activation by affecting the assembly and activity of the protein kinase Czeta/p62/TRAF6 signaling complex. Mol Cell Biol 25: 4010-4022, 2005.

24. Deng YH, Alex D, Huang HQ, Wang N, Yu N, Wang YT, Leung GP and Lee SM: Inhibition of TNF- $\alpha$-mediated endothelial cell-monocyte cell adhesion and adhesion molecules expression by the resveratrol derivative, trans-3,5,4'-trimethoxystilbene. Phytother Res 25: 451-457, 2011.

25. Bonnefont-Rousselot D: Resveratrol and cardiovascular diseases. Nutrients 8: 250, 2016 